\title{
Uber die Beeinflussung der Glykolyse von Ehrlich-Ascites-Tumorzellen durch phosphorylierte Fructosen
}

\author{
Von E. Heise, W. Lührs und M. Görlich \\ Aus der Robert-Rössle-Klinik des Institutes für Medizin und Biologie \\ der Deutschen Akademie der Wissenschaften zu Berlin \\ (Z. Naturforschg. 16 b, 582—585 [1961] ; eingegangen am 18. März 1961)
}

\begin{abstract}
Fructose-1.6-diphosphat kann bei Tumor-Ascites-Zellen im glucosefreien Medium eine Glykolyse hervorrufen, die nur ca. $20 \%$ der Glykolyse unter normalen Bedingungen beträgt.

Die Milchsäurebildung verläuft bei geringen FDP-Konzentrationen proportional der Substratmenge. Höhere Konzentrationen als ca. $19 \mu$ Mole FDP steigern die Glykolyse nicht weiter.

Enzymatisch hergestelltes Fructose-6-phosphat ruft eine größere Glykolyse als entsprechende FDP. Konzentrationen hervor. Dies scheint auf einer besseren Penetrationsfähigkeit des F6P durch die Zellmembran zu beruhen.

Zusatz von Insulin steigert die durch FDP induzierte Milchsäurebildung um ca. 100 Prozent.
\end{abstract}

Wie wir kürzlich mitteilten ${ }^{1}$, ist es möglich, die anaerobe und aerobe Glykolyse von Ehrlich-AscitesKarzinom-Zellen durch Zusatz von aus Rinderleber isolierter und angereicherter Fructose-1.6-Diphosphatase (FDPase $)^{2}$ um $60 \%$ zu hemmen. Durch $\mathrm{Zu}$ satz der FDPase wird eine bei Tumorzellen sonst einseitig verlaufende Reaktion, die Phosphorylierung von Fructose-6-Phosphat (F6P) zu Fructose1.6-Diphosphat (FDP) in die reversible Richtung der Dephosphorylierung gedrängt, wodurch die Spaltung von Glucose zu Milchsäure gestört wird. Dieser Befund wäre einleuchtend, wenn wir in Homogenaten oder mit reinem Cytoplasma gearbeitet hätten. Unsere Untersuchungen wurden jedoch mit intakten, vitalen Ehrlich-Ascites-Karzinom-Zellen durchgeführt. Da nach Frunder und Mitarbb. ${ }^{3}$ die Membran von Ascites-Zellen für phosphorylierte Hexosen nicht durchlässig ist, waren unsere Versuchsergebnisse, die immer wieder reproduzierbar waren, eigentlich nicht zu erwarten.

Zur Klärung des Mechanismus der FDPase-Wirkung haben wir deshalb Untersuchungen über die Durchlässigkeit von Ascites-Zell-Membranen für FDP und Fructose-6-Phosphat durchgeführt.

\section{Materialien und Methoden}

Sämtliche Versuche über die anaerobe Glykolyse wurden mit Ehrlich-Ascites-Karzinom-Zellen durchgeführt. Als Inkubationsmedium diente entweder ein

1 W. Lührs, E. Heise u. M. Görlich, Z. Naturforschg. 16 b, 254 [1961].

2 K. Lohmans, Biochem. Z. 262, 137 [1933].

3 N. Sönnichens, H. Frunder, H. Börnig u. G. Richter, HoppeSeyler's Z. physiol. Chem. 316, 209 [1959].
Gemisch aus Ringe r-Lösung, Natriumbicarbonat und Glucose bzw. aus R i n g e r - Lösung und Natriumbicarbonat in den substratfreien Versuchen oder ein Gemisch aus Phosphatpuffer $\left(0,1-m\right.$. und $\left.p_{\mathrm{H}} 7,4\right)$ und Glucose bzw. nur Phosphatpuffer für andere substratfreie Versuche. Die Ascites-Zellen wurden in W a r b u r g-Gefäßen mit einem Anhang 60 Min. lang bei $37{ }^{\circ} \mathrm{C}$ inkubiert. Der Gasraum enthielt 95\% Stickstoff und 5\% Kohlendioxyd. Der Anhang der Gefäße enthielt $0,1 \mathrm{ml} 20$-proz. Trichloressigsäure (TCE) zur Unterbrechung des Stoffwechsels der Ascites-Zellen. Die gebildete Milchsäure wurde chemisch nach der Methode von Barker und Summerson ${ }^{4}$ bestimmt. Jede Versuchsanordnung bestand aus zwei Gefäßen mit gleichem Inhalt. Der Stoffwechsel des Gefäßes 1 wurde zur Zeit 0 durch Einkippen der TCE gestoppt, der Stoffwechsel des zweiten Gefäßes nach genau 60 Minuten.

Die Differenz des gebildeten Lactats in beiden Gefäßen ergibt die Milchsäurebildung durch die AscitesZellen innerhalb einer Stunde. Die Phosphatbestimmungen erfolgten nach der von $\mathrm{F}_{\text {ISKe }}$ und Subbarow ${ }^{5}$ angegebenen Methode. F6P und FDP wurden als handelsübliche Präparate der Firma Boehringer \& Söhne angewandt.

\section{Versuchsanordnungen und Versuchsergebnisse}

In den ersten Versuchen wurden Ascites-Zellen in einem R inge r - Bicarbonat-Glucose-Gemisch (RBG) bzw. in einem substratfreien Gemisch ohne Glucose inkubiert. Außerdem wurde in weiteren Versuchen die Glucosemenge $(5,4 \mathrm{mg} / \mathrm{Gefäßinhalt)}$ durch die entsprechende Menge FDP (11,3 mg) bzw. ganzer Vielfacher davon ersetzt. Die FDP-Mengen wurden genau abgewogen, in einem Ringer-Bicarbonat-Gemisch gelöst und zu den Ascites-Zellen hinzugegeben.

\footnotetext{
4 S. B. Barker u. W. H. Summerson, J. biol. Chemistry 138 , 535 [1941].

5 C. H.Fiske u. Y.Subbarow, Methods in Enzymology, Bd.III, S. $843,1957$.
} 
Wie die Abb. 1 zeigt, kann in einem substratfreien Milieu keine Milchsäurebildung nachgewiesen werden. Wenn man aber auch Glucose durch FDP ersetzt, wird Milchsäure gebildet. Diese Milchsäurebildung beträgt jedoch nur $20-25 \%$ der durch die entsprechende Menge Glucose hervorgerufenen Glykolyse.

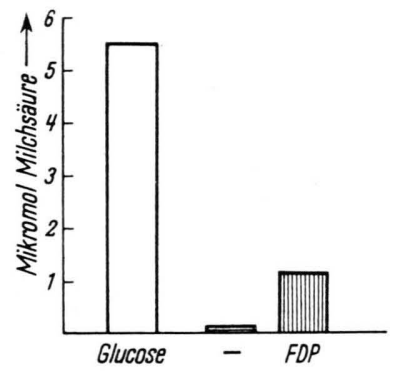

Abb. 1. Anaerobe Glykolyse von $1 \mathrm{mg}$ Ascites-Zellen in R i n g e r - Bicarbonat-Puffer, $\mathrm{pH}_{\mathrm{H}} 7,4 \square$ mit 3,4 $\mu$ Molen Glucose $\square$ ohne Substrat $\square$ mit $18,0 \mu$ Molen FDP.

Aus der Abb. 2 ist der Einfluß steigender FDPKonzentrationen auf die Glykolyse zu ersehen. Es zeigte sich, daß bis zu einem bestimmten Grenzwert die FDP-Konzentrationen der Milchsäurebildung proportional verlaufen. Nach Überschreiten der Grenzkonzentration kann auch durch weitere erhöhte FDP-Konzentrationen keine zusätzliche Steigerung der Milchsäurebildung erreicht werden.

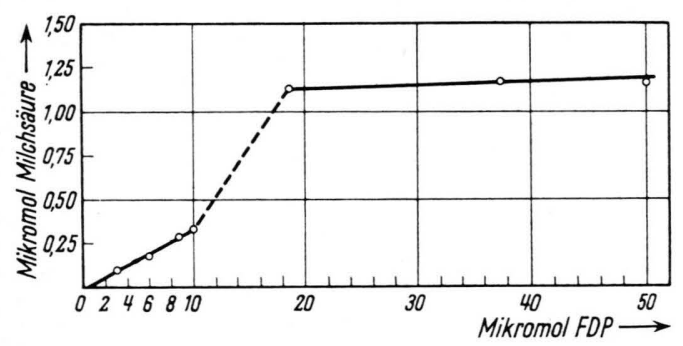

Abb. 2. Einfluß steigender FDP-Konzentrationen auf die anaerobe Glykolyse von $1 \mathrm{mg}$ Ascites-Zellen in $\mathrm{R}$ i n $\mathrm{g}$ e $\mathrm{r}$ Bicarbonat-Puffer, $p_{\mathrm{H}} 7,4$.

Offensichtlich ist die Durchlässigkeit der Membran von Ascites-Zellen bei dieser bestimmten FDP-Konzentration erschöpft. Auf Grund ähnlicher Versuche kamen auch Tiedemann und Bors ${ }^{6}$ zu dem Schluß, daß phosphorylierte Hexosen nur sehr langsam die Zellmembran durchdringen können.

6 H. Tiedemann u. J. Born, Hoppe-Seyler's Z. physiol. Chem. 321, 205 [1960].
Die Abb. 3 zeigt weitere Ergebnisse folgender Versuchsanordnungen:

Gegenüber der durch RBG hervorgerufenen Glykolyse war erwartungsgemä $\beta$ bei Verwendung von Phosphatpuffer als Inkubationsmedium und Glucose als Substrat eine Steigerung der Milchsäurebildung zu beobachten.

Im substratfreien Medium war keine Glykolyse nachzuweisen, während durch Zusatz von FDP als Substrat eine Milchsäurebildung hervorgerufen wird. Sie beträgt aber nur $25 \%$ der durch die gleiche FDP-Konzentration in RB hervorgerufenen Glykolyse.

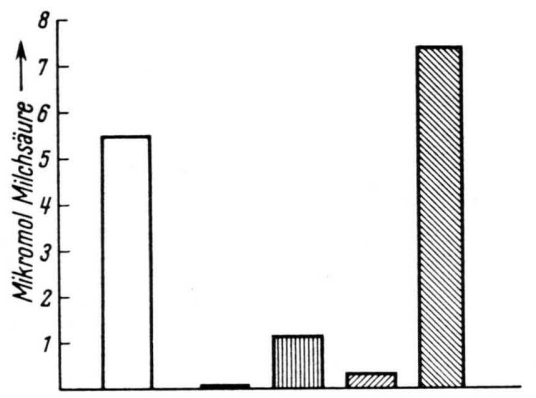

Abb. 3. Anaerobe Glykolyse von $1 \mathrm{mg}$ Ascites-Zellen. $\square$ in $\mathrm{R}$ in g e r - Bicarbonat-Puffer mit 3,4 $\mu$ Molen Glucose, $\square$ in Phosphatpuffer ohne Substrat, $\square$ in $\mathrm{R}$ i n g e r - BicarbonatPuffer mit 18,0 $\mu$ Molen FDP, WIII in Phosphatpuffer mit 18,0 $\mu$ Molen FDP, in Phosphatpuffer mit 3,4 $\mu$ Molen Glucose.

Diese Versuchsergebnisse lassen vermuten, daß FDP an den Zellmembranen zuerst dephosphoryliert werden muß, um in die Zellen, vielleicht auch durch Wiederaufnahme von Phosphat, eindringen zu können.

Durch Zusatz von anorganischem Phosphat würde die Dephosphorylierungsreaktion zurückgedrängt, und der Eintritt von FDP in die Zellen verhindert werden. Wenn diese Hypothese richtig wäre, müßte es möglich sein, durch Fructose-6-Phosphat (F6P) eine Stimulierung der Milchsäurebildung gegenüber FDP zu erreichen. Da uns F6P nicht zugänglich war, haben wir FDP mit FDPase in der bereits beschriebenen Weise ${ }^{1}$ inkubiert, um auf enzymatischem Wege F6P herzustellen. Sofort nach der Inkubation wurde 15 Min. lang auf $60^{\circ}$ erhitzt, um die Wirkung der FDPase abzustoppen. Nach dem Zentrifugieren konnte der klare Überstand als eine F6P enthaltende Lösung verwendet werden. 
Abb. 4 zeigt den Einfluß steigender FDP-Konzentrationen, die vorinkubiert wurden, auf die Milchsäurebildung. Aus den Ergebnissen ist zu ersehen, daß zur Bildung größerer Mengen Milchsäure bei Vorinkubation weniger FDP erforderlich war, als ohne Vorinkubation.

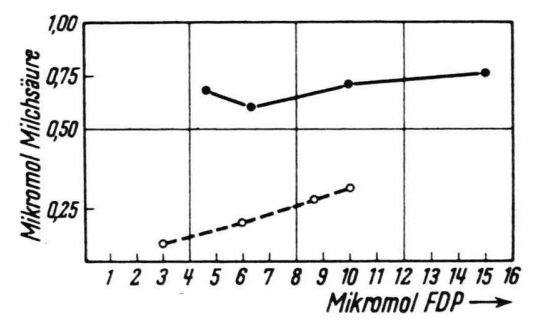

Abb. 4. Anaerobe Glykolyse von $1 \mathrm{mg}$ Ascites-Zellen. - FDP mit FDPase vorinkubiert, $0--0$ FDP.

Da bei allen FDP-Konzentrationen gleiche FDPase-Mengen verwendet wurden, war eine gleich große Menge F6P zu erwarten. Diese F6P-Konzentration ruft, wie aus der Abb. 4 ersichtlich ist, stets eine gleich große Glykolyse hervor. Die spezifische Aktivität der verwendeten FDPase betrug 0,957.

Die FDPase-Lösung enthielt 39,5 mg Protein pro $1 \mathrm{ml}$. Zur Inkubation gelangten $0,2 \mathrm{ml}=7,9 \mathrm{mg}$ Protein. Die Inkubationsdauer betrug 45 Min.; dabei wurden 5,7 $\mu$ Mole F6P gebildet.

Von dieser Lösung wurde $1 / 4$ pro Gefäß inkubiert, d. h. ca. 1,4 $\mu$ Mole F6B wurden mit Ascites-Zellen inkubiert. Die durch diese F6P-Menge hervorgerufene Glykolyse ist viel größer als die durch die entsprechende Menge FDP hervorgerufene Milchsäurebildung. Allerdings befindet sich in der Inkubationslösung neben dem F6P noch das restliche FDP, das bekanntlich ebenfalls eine schwache Glykolyse her- vorruft. Aus diesem Grunde überlagern sich beide Effekte, so daß eine einwandfreie Aussage nicht möglich ist. Wir haben deshalb den Verbrauch an Phosphat während der Inkubation bestimmt. AscitesZellen wurden wie beschrieben, mit FDP bzw. mit enzymatisch hergestelltem F6P 60 Min. lang im Thermostaten inkubiert. Nach Fällung mit TCE wurde zentrifugiert und $1 \mathrm{ml}$ des Überstandes mit $0,2 \mathrm{ml} \mathrm{HClO}_{4}$ verascht. Der Rückstand wurde in $1 \mathrm{ml}$ Wasser aufgenommen und davon $0,1 \mathrm{ml}$ zur Phosphatbestimmung verwendet. Die folgende Tab. 1 zeigt den Phosphatverbrauch durch $1 \mathrm{mg}$ AscitesZellen mit und ohne Vorinkubation des zugesetzten FDP.

Aus der Tabelle ist ersichtlich, daß bei Vorinkubation mehr Phosphat verschwindet als ohne Vorinkubation. Die verschwundenen Phosphatmengen sind jedoch größer als die durch die FDPase aus FDP freigesetzten Phosphatmengen. Es muß folglich F6P in die Zellen eingetreten sein. In der letzten Spalte sind die mit F6P bzw. FDP zusammen in die Zelle eingetretenen Phosphatmengen angeführt. Es zeigt sich, daß mehr Phosphat in Form von F6P als in Form von FDP von den Zellen aufgenommen wird. Folglich tritt mehr F6P als FDP in die Zellen ein. Die bei Verwendung von F6P beobachteten höheren Milchsäuremengen sind auf eine bessere Penetration von F6P durch die Zellmembran zurückzuführen.

Allerdings könnte es möglich sein, daß FDP beim Inkubieren bei $38{ }^{\circ} \mathrm{C}$ bzw. in der Zeit, in der die Ascites-Zellen vorbereitet werden, einer Zersetzung anheimfällt und Phosphat abspaltet. Wir haben deshalb FDP in RB verschiedene Zeiten bei $38{ }^{\circ} \mathrm{C}$ inkubiert und die während dieser Zeit abgespaltenen Phosphatmengen bestimmt.

\begin{tabular}{|c|c|c|c|c|c|}
\hline $\begin{array}{c}\text { Zugesetztes } \\
\text { FDP } \\
{[\mu \text { Mole }]}\end{array}$ & $\begin{array}{l}\text { Ascites } \\
{[\mathrm{mg}]}\end{array}$ & $\begin{array}{l}\text { Milchsäurebildung } \\
{[\mu \mathrm{Mol} / \mathrm{mg} \text { Ascites] }}\end{array}$ & $\begin{array}{c}\text { Phosphat- } \\
\text { Verbrauch } \\
{[\mu \mathrm{Mol} / \mathrm{mg} \text { Ascites] }}\end{array}$ & $\begin{array}{c}\mathrm{PO}_{4} \text { durch FDPase } \\
\text { freigesetzt } \\
{[\mu \mathrm{Mol}]}\end{array}$ & $\begin{array}{c}\text { Als FDP bzw. F6P } \\
\text { in die Zellen } \\
\text { eingetretenes } \\
\mathrm{PO}_{4}[\mu \mathrm{Mol}]\end{array}$ \\
\hline 3,0 & 6,7 & 0,084 & - & - & - \\
\hline 5,9 & 6,4 & 0,17 & 0,2 & - & 0,2 \\
\hline 8,7 & 6,4 & 0,28 & 0,3 & - & 0,3 \\
\hline 24,6 & 2,4 & 0,34 & 0,39 & - & 0,39 \\
\hline 18,7 & 2,4 & 1,1 & - & - & - \\
\hline 37,4 & 2,4 & 1,1 & - & - & - \\
\hline $4.7 *$ & 2,1 & 0.67 & 2,20 & 0,67 & 1,53 \\
\hline $6,3^{*}$ & 2,1 & 0,58 & 1,83 & 0,67 & 1,16 \\
\hline $10,0^{*}$ & 2,1 & 0,70 & 2,00 & 0,67 & 1,33 \\
\hline
\end{tabular}

Tab. 1. Phosphatverbrauch durch $1 \mathrm{mg}$ Ascites-Zellen mit und ohne Vorinkubation des zugesetzten FDP. Mit FDPase vorinkubiert. 
Aus der Abb. 5 ist ersichtlich, daß die während 90 Min. abgespaltenen Phosphatmengen äußerst gering sind und in keinem Verhältnis zu den durch die Ascites-Zellen abgespaltenen Mengen stehen.

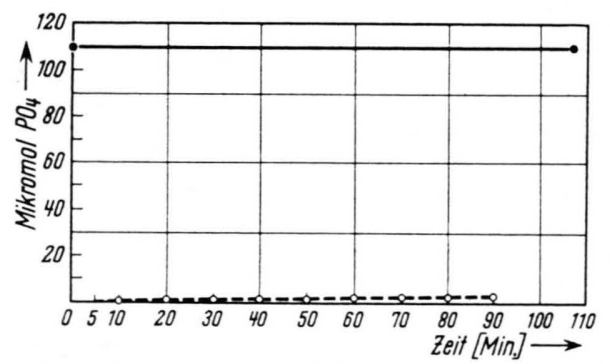

Abb. 5. $\mathrm{PO}_{4}$-Abspaltung aus FDP in verschiedenen Zeiten. -— eingesetzte $\mathrm{PO}_{4}$-Menge im FDP, $0--0$ während der Inkubation abgespaltene $\mathrm{PO}_{4}$-Mengen.

Es ist bekannt ${ }^{\text {a u. b. }}$, daß durch Insulin die Glykolyse gesteigert werden kann. Diese Erscheinung ist auf eine stimulierende Beeinflussung der Hexokinase durch Insulin zurückzuführen. Die bei unseren Versuchen durch Zusatz von FDP zu glucosefreien Ascites-Zellen beobachtete Glykolyse schließt die Hexokinasereaktion aus. In diesem Fall dürfte Insulin keinen Einfluß auf die Milchsäurebildung haben. Unsere Versuche zeigten jedoch das Gegenteil.

Vielleicht kann diese Wirkung durch die Annahme erklärt werden, daß Insulin die Durchlässigkeit der Zellmembran für FDP steigert, ähnlich wie es FrUnder $^{3}$ für Fructose-6-Phosphat an Leberversuchen gezeigt hat.

7 W. C. Stadie, Physiol. Rev. 34, 52 [1954].

7 Ch. De Duve, in: 5. Coll. d. Ges. f. physiol. Chem. Mosbach/Baden 1954, S. 108, Berlin, Göttingen u. Heidelberg 1955.

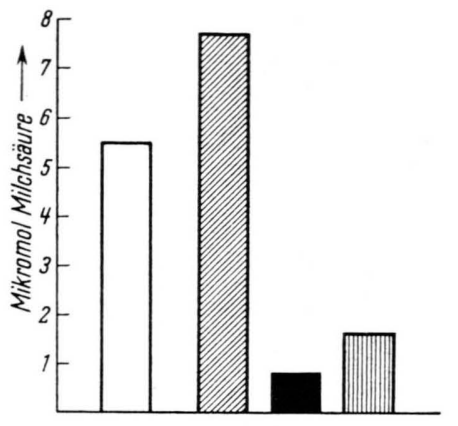

Abb. 6. Anaerobe Glykolyse von $1 \mathrm{mg}$ Ascites-Zellen. [III in R in g e r - Bicarbonat-Glucose + 0,5 Einheiten Insulin, $\square$ in $\mathrm{R}$ in g e $\mathrm{r}$ - Bicarbonat-FDP + 0,5 Einheiten Insulin, in $\mathrm{R}$ in g e r - Bicarbonat-Glucose, $\square$ in $\mathrm{R}$ in g e r Bicarbonat-FDP.

\section{Diskussion}

Es kann als sicher angenommen werden, daß FDPase die Membran von Ascites-Zellen nicht durchdringen kann. Trotzdem kann das Enzym die Glykolyse von intakten Ascites-Zellen herabsetzen. Diese Tatsache ist nur durch die Annahme zu erklären, daß das Fermentsubstrat (FDP) die Zellwand passieren kann. FDP könnte dann im extrazellulären Raum durch FDPase gespalten werden und als F6P wieder in die Zellen eintreten. Wir haben versucht, in der vorliegenden Arbeit den Beweis zu erbringen, daß sowohl FDP als auch F6P die Membran von Ascites-Zellen passieren können. Allerdings ist diese Durchdringungsfähigkeit sehr klein, aber für F6P größer als für FDP. Da jedoch ein dauernder Austausch zwischen Zellplasma und extrazellulärem Raum möglich ist, könnte die Wirkung der FDPase auf diese Weise erklärt werden. 